\title{
Missing link - Risiken des Sparens
}

\author{
Christof Schmitz ${ }^{a, d}$, Marcel Zwahlen ${ }^{b, e}$, Peter Berchtold ${ }^{c, d, e}$ \\ ${ }^{a}$ Dr.; ${ }^{b}$ Prof. Dr.; ${ }^{\text {P PD Dr.; }}$ college M, Bern; ${ }^{e}$ Institut für Sozial- und Präventivmedizin, Universität Bern
}

\begin{abstract}
Der ökonomische Druck zwingt viele Spitäler zu intensivierten Versuchen der Effizienzgewinnung, wenn nicht gleich zu Sparmassnahmen. Ein Risiko besteht: Steigerungen der Effizienz können in Effektivitätsverluste kippen, z.B. wenn gesenkte Kosten die Qualität der Patientenbetreuung gefährden. Will man diesen Zusammenhang näher verstehen, zeigen sich die Verbindungen zwischen Medizin und Ökonomie als erstaunlich unklar, sieht man von Binsenweisheiten wie «eine vernünftige Medizin mit guten Prozessen macht sich sicher bezahlt» ab. Wir schlagen vor, verstärkt in «Loops» zu denken, um dem missing link zwischen Effizienz und Effektivität auf die Spur zu kommen.
\end{abstract}

2018 war ein wenig erfreuliches Jahr für viele Spitäler. Die "neue Runde», die eingeläutet ist, drückt sich in vielfach unbefriedigenden Ergebnissen aus [1]. Viele Häuser sind entsprechend von Sparmassnahmen, Kostenreduktionsprogrammen und sonstigen Überlegungen zu EBITDA- und Effizienzsteigerung erfasst. Das sorgt für Unruhe in den klinischen Bereichen, fühlt

Wie immer in komplexen Verhältnissen haben beide Seiten so recht wie unrecht und der Streit ums Rechthaben führt nirgendwohin.

man sich dort teilweise "ausgepresst wie eine Zitrone» (Zitat eines Chefarztes). Die Spannung ist eine doppelte: Auf der einen Seite erhöht sich der Druck, effizienter zu werden. Dies umso mehr, als die Wachstumsstrategien der letzten Jahre nicht mehr gleichermassen

\section{Résumé}

La pression économique oblige de nombreux hôpitaux à intensifier leurs efforts de gain d'efficience, voire à mettre en œuvre des mesures d'économies. Cela comporte un risque: I'accroissement de l'efficience peut basculer vers des pertes d'efficacité, lorsque la baisse des coûts compromet la qualité de la prise en charge des patients, par exemple. Si l'on cherche à mieux comprendre ce phénomène, les liens entre la médecine et l'économie s'avèrent étonnamment flous, au-delà d'évidences telles que: "une médecine raisonnable avec des processus appropriés est certainement avantageuse." Nous suggérons de raisonner davantage en "boucles» pour mettre au jour le chaînon manquant entre l'efficience et l'efficacité. greifen. Auf der anderen Seite zerren neben dieser Aussenspannung auch Innenspannungen an den Nerven, insbesondere jene zwischen Management und Kerngeschäft. Ersteres nimmt typischerweise an, dass noch «Luft im System», ist und versucht die Kaderärzte mit Kaskaden von Key Performance Indicators zu, nun ja, motivieren, während Letzteres meint, dass bald der letzte Tropfen ausgequetscht sein wird, und sich fragt, ob die "Controller» nicht zunächst an sich selbst sparen sollten. Wie immer in komplexen Verhältnissen haben beide Seiten so recht wie unrecht und der Streit ums Rechthaben führt nirgendwohin. Stattdessen wäre zu fragen: Was wird übersehen in dieser unfruchtbaren Konfrontation?

\section{Missing link}

Vor einiger Zeit konnten wir in Deutschland, das in Bezug auf die Konfliktschärfe zwischen Management und Medizin deutlich - und nicht mit Vorteil - vor der Schweiz liegt, einer Diskussion zwischen einem Hotelmanager und Ärzten und Ärztinnen beiwohnen. Nach einer Runde heftiger Klagen der Mediziner über das Unverständnis und die Ignoranz ihrer Geschäftsführungen fragte der Hotelmanager: «Warum sagen Sie Ihren Geschäftsführern nicht einfach, was Sie brauchen?» Fassungsloses Schweigen in der Runde, dann die Frage, was er denn meinen würde. Der Hotelier schilderte, wie bei ihnen, einer Hotelkette im oberen Preissegment, alle Prozesse definiert und viele auch mit Zeitangaben versehen wären. So könne man beispielsweise gut argumentieren, dass, wenn 100 Gäste 
pro Tag einchecken sollen (eine Managementvorgabe), so und so viel Personalkapazität gegeben sein muss, man wisse ja schliesslich, wie viele Minuten ein durchschnittliches Check-in dauern würde. So seien lösungsorientierte Diskussionen möglich. Ob das in den Krankenhäusern anders sei? Schweigen in der Runde.

Diese kleine Unterhaltung beleuchtet einen interessanten Punkt, nämlich, wie Ressourcenbedarf und Leistungserbringung zusammengedacht werden. Das

Warum sagen Sie Ihren Geschäftsführern nicht einfach, was Sie brauchen?

Thema gewinnt unmittelbare Relevanz, wenn Sparen angesagt ist und es zu diskutieren gilt, was beschnitten oder wo eingegrenzt werden soll - und welche Auswirkungen das kurz- als auch langfristig haben wird. Klar, wir wissen, dass es bestimmte Personalbestände braucht, um Vorhalteleistungen oder Dienstnotwendigkeiten zu erfüllen. Wir wissen, dass eine chirurgische Klinik oder ein Tumorcenter mehr ist als das Genius des Chefs und aus verschiedenen Puzzlestücken besteht, die gut zueinander passen müssen. Aber ist auch hinreichend klar, was diese Puzzlestücke ausmacht und wie die Qualität ihres Zusammenspiels zum Gelingen oder Misslingen des medizinischen wie ökonomischen Erfolgs beiträgt? Ist hinreichend verständlich, was effektiv ist und was den Erfolg einer Klinik im Sinne ihres gesamthaften Funktionierens ausmacht? Ansätze existieren, z.B. liefern Zertifizierungsanforderungen wichtige Hinweise. Solche Überlegungen beschränken sich jedoch das ist auch ihre Absicht - auf medizinische Zusammenhänge und sind losgelöst von anderen Faktoren, etwa der Wirtschaftlichkeit, der Zufriedenheit der Mitarbeitenden oder auch der Patientennutzen. Dieser missing link erschwert rationale Diskussionen und Entscheidungen über Effizienzsteigerungen zwischen Management und Klinikern, weil (Miss-)Erfolgsbedingungen effektiv im Unklaren bleiben.

\section{Ein erster Loop}

Eine zweite Unterhaltung: Eine Ärztin, die kürzlich in einen nichtklinischen Bereich gewechselt war, erzählte, dass sie mit dem Wechsel beschlossen hatte, Selbstmanagementtools anzuwenden. Bereits mit der Erstellung einer einfachen To-do-Liste wurde ihr klar, dass das, was sie zu tun hatte, mit der vorhandenen Kapazität gar nicht zu leisten ist. Mit dieser Erkenntnis ging sie zu ihren Vorgesetzten und die - siehe da! liessen sich überzeugen und kümmerten sich in der Folge um Kapazitätsausbau. So weit, so erfolgreich.
Dann meinte die Ärztin noch, dass sie in ihrer klinischen Zeit mit derselben Erkenntnis niemals zu ihrem Chef gegangen wäre. Denn der hätte höchstens gemeint: Du musst dich besser organisieren! Allgemeine Zustimmung in der Runde der zuhörenden Mediziner. Nicht nur zwischen Management und Kliniken, auch innerhalb klinischer Leistungseinheiten findet sich oftmals begrenzte Klarheit darüber, wie und wie viel von wem und in welchem Zusammenspiel leistbar ist und wo Kipppunkte für Erfolg bzw. Misserfolg liegen. Ein Grund dafür liegt in der vielfach beobachtbaren (sozialpsychologisch sehr bekannten) Attributionspraxis, Belastungen und Misslingen eher Personen - «Du musst dich besser organisieren!» - und nicht Situationen (Organisation, Struktur, Kapazitäten etc.) zuzuschreiben. Das gilt in besonderem Masse unter Ärzten und hat vermutlich mit der tief sitzenden professionellen Idee zu tun, persönliche Kompetenz mache den Unterschied aus.

Exemplarisch: Eine Klinik kommt leistungsmässig unter Druck. Die Zahlen schwächeln. Der Chef wird nervös und kritisiert seine jungen Oberärzte und -ärztinnen. Die finden das ungerecht. Sie ärgern sich, was ihre Leistung eher senkt und die Personalfluktuation erhöht, was der Arbeitsmarkt locker zulässt. Das Management beobachtet das, wird seinerseits noch unruhiger und fordert den Chefarzt auf, seine Klinik endlich

\section{Eine Spirale, ein Loop fehlgehender Zuschrei-} bungen ist in Gang gekommen, und der kann nun richtig Fahrt aufnehmen.

in den Griff zu kriegen. Das macht den Chef nochmals gereizter, er beanstandet noch intensiver ... der allgemeine Stresspegel steigt weiter. Eine Spirale, ein Loop fehlgehender Zuschreibungen ist in Gang gekommen, und der kann nun richtig Fahrt aufnehmen. Ein Teufelskreis bildet sich: Steigender Stress führt zu $\mathrm{Zu}-$ schreibung auf Personen ("Die sind nicht kompetent») und aktualisiert in der Folge stereotypische Vorurteile: des Managements gegenüber dem Chefarzt («Der kann nicht führen»), dem Chefarzt gegenüber dem klinischen Nachwuchs («Die taugen nichts heute»), dem klinischen Nachwuchs gegenüber dem Chefarzt («Der kümmert sich nicht um uns») etc. Diese Aktualisierung typischer Vorurteile und der sie begleitenden negativen Konnotierungen unterminiert die Chance, miteinander zu klären, wie die Klinik erfolgreich werden könnte und was die Bedingungen dafür wären. Mit anderen Worten: Erhöhter Stress im System senkt die Wahrscheinlichkeit, dass sinnvolle Massnahmen der Verbesserung identifiziert und gewählt werden (siehe Abb. 1: Stressloop). 


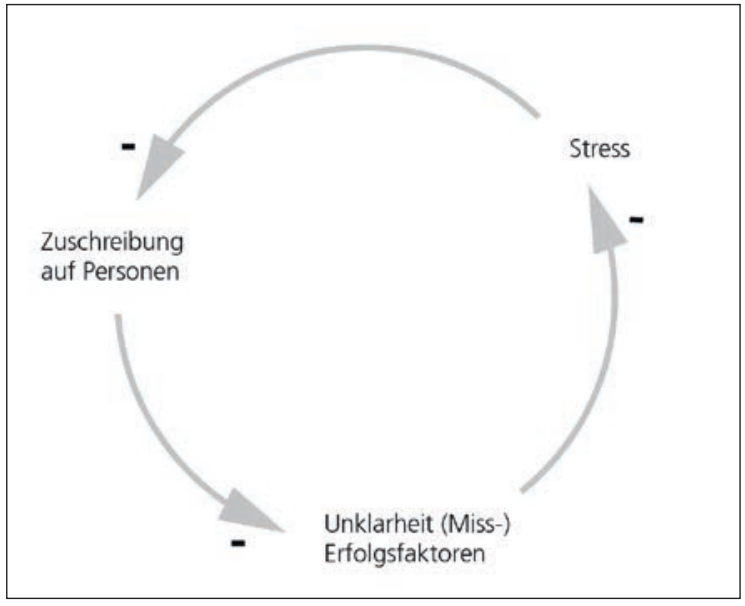

Abbildung 1: Stressloop (eigene Darstellung).

\section{Teufelskreise}

Einen Vorschlag von Schwaninger und Klocker (2018) aufgreifend postulieren wir folgenden zweiten Zusammenhang: Klinische Leistungserbringung ist wesentlich durch die Wechselwirkung zwischen Arbeitslast (patient load), Stress des Personals sowie Qualität der Behandlung gekennzeichnet (siehe Abb. 2: Qualitätsloop).

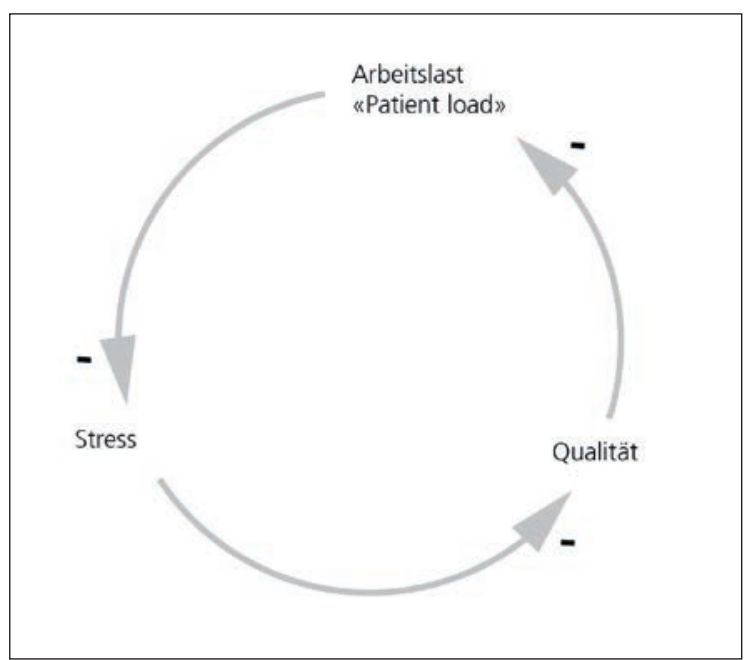

Abbildung 2: Qualitätsloop (adaptiert nach [2]).

Folgende Rückkopplung kann beobachtet werden: Steigende Last (sei es in quantitativer wie qualitativer Hinsicht) treibt den Stresspegel des Personals nach oben. Letzterer ist wesentlich vom Kompetenzgrad des Personals abhängig. Offensichtlich kann kompetentes Personal eine höhere «Last» bewältigen als unerfahrenes. Erhöhter Stress führt nun zu abnehmender Qualität der Behandlung, was wiederum zu einer Steigerung der «Last» beiträgt (via längere Behandlungszeiten, Komplikationen, Beschwerden, weitere Unzufriedenheiten) - und der Teufelskreis beginnt seine Wirkung zu entfalten.

Diesen Teufelskreis kennt man aus dem klinischen Alltag. Stress steigt, weil die Personaldecke dünn wird. Erfahrenes Personal wird unzufrieden, weil zusehends gestresst. Es beschwert sich und wird nicht gehört (siehe Abbildung 1: Stressloop). Ärger und Friktionen breiten sich aus. Die Leitung findet, es habe «noch Luft im System", und verschliesst sich der eigentlichen Dynamik. Schliesslich suchen die kompetenten Leute das Weite. Weniger erfahrenes Personal wird eingestellt und arbeitet langsamer und aufwendiger. Damit erhöht sich die Arbeitslast, was erneut den Stresspegel steigen lässt und die Behandlungsqualität senkt. Die Folge sind Kritik in der Klinik, verstärkter Druck auf das ärztliche Personal, gefolgt von weiteren Personalwechseln mit nochmals sinkender Behandlungsqualität. Rasch beginnt sich das herumzusprechen, die Zuweiser werden aufmerksam, das Management macht sich Sorgen und droht mit Budgetkürzungen und weiter dreht die Abwärtsspirale.

Darum können Sparmassnahmen prekär sein. Nicht weil sie den einen kleinen Luxus oder das andere Niceto-have kappen würden. Das kann (quasibiologisch gesprochen) sogar die Fitness erhöhen. Prekär sind sie, wenn sie Teufelskreise auszulösen drohen. Dann ergibt (wenn wir von einer gut funktionierenden Klinik ausgehen) die Budgetsenkung kurzfristig eine zunächst erfreulich erscheinende Kostendämpfung, aber bereits mittelfristig wird die Qualität und damit die Essenz, um die es geht, bedroht [2]. Die Folgen können zerstörerisch sein, ein mechanism of desaster greift Platz. Dann vegetieren Kliniken dahin auf niedrigem Niveau mit mässiger Qualität, getragen von einer gewissen Ausbeutungsbereitschaft des ärztlichen Personals, aber fortlaufend bedroht, zusammenzubrechen. Das ist das Risiko des Sparens: Zugunsten kurzfristiger Effizienz kann mittelfristig Effektivität zerstört werden.

\section{Dem missing link auf der Spur}

Die beiden Loops sind via «Stress» verbunden (siehe Abbildung 3: Der Doppelloop). Kritischer Weise drohen beide Loops "hochzudrehen» und den Stress doppelt zu verstärken. Die Möglichkeit bestünde jedoch, via Stressloop zur Beruhigung beizutragen. Dann muss die Attributionspraxis geändert werden und einer differenzierten Auseinandersetzung, was organisatorisch Erfolg ausmacht, Platz machen. Loops können ja auch nach oben weisen. Ein Beispiel: Alle informierten Männer mit Prostataerkrankungen wollen heute in die 


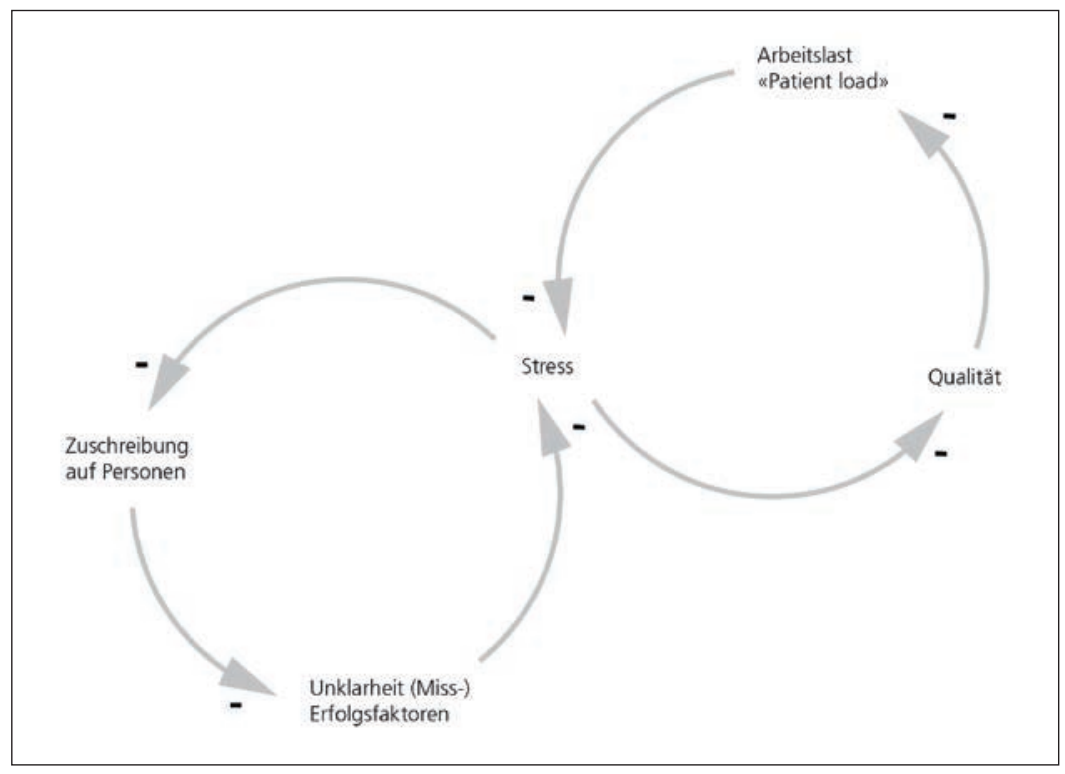

Abbildung 3: Der Doppelloop (adaptiert nach [2]).
Practice-Übertragung. Aber die Klarheit über das eigene Erfolgsmodell (drei Prinzipien) besticht und inspiriert. Und eindrücklich $\mathrm{zu}$ sehen, dass das Hamburger Modell den Weg über Patient-reported Outcome Measures (PROMs) geht, also über eine erweiterte Qualitätsmessung. Braucht es eventuell genau dieses Feedback des erweiterten Patientenwohlergehens, um über Effektivität entscheiden zu können? Können wir hier ein Mittel finden, um Klarheit über Erfolg zu gewinnen? Ist hier der entscheidende Hinweis zum missing link zu finden? Value Based Healthcare tendiert in diese Richtung. Das mag noch nicht die ganze Wahrheit sein, aber in eine essenzielle Richtung weisen.

Eigentlich ist es ja merkwürdig. Irgendwie scheinen diese Modelle bislang wenig relevant gewesen zu sein. Stattdessen werden Vorurteile und Stress gepflegt und teure Berater angeheuert, die Daten um Daten erheben, damit das Management beurteilen kann, wie viel die Zitrone noch gepresst werden kann, und dann doch immer wieder neu entdecken muss, dass die vermeintlich scharfen Analysen den Nebel wenig zu lichten wissen. Daten alleine sprechen bekanntlich noch nicht. Entscheidend ist: Werden die richtigen Fragen gestellt? Was helfen Marktanalysen, wenn unklar bleibt, was die Systembedingungen für Effektivität sind? Würde diesbezüglich mehr Klarheit herrschen, könnten Massnahmen der Effizienzsteigerung präziser von Effektivitätsgefährdungen unterschieden werden. Das wäre zum Wohle der Patienten als auch des Personals.

\section{Literatur}

1 Schmitz C, Zwahlen M, Berchtold P. Das Management der Medizin. Schweiz Ärzteztg. 2018;99(33):1078-80.

2 Schwaninger M, Klocker J. Efficiency Versus Effectiveness in Hospitals: A Dynamic Simulation Approach. In Borgonovi E, Anessi-Pessina E, Bianchi C, editors. Outcome-Based Performance Management in the Public Sector. Springer Nature; 2018.
Dr. Christof Schmit college M

Haus der Akademien Laupenstrasse 7 CH-8001 Bern
Klinik weiter herumspricht usf. Eine Aufwärtsspirale ist in Gang gesetzt. Manch einer wird jetzt einwenden, das Prostatakarzinom sei ein «Nischenprodukt» und nicht vergleichbar mit der Anforderung, mit breitgefächerten Diagnosen einen Umgang zu finden. Klar gibt es grosse Unterschiede und keine einfache Best-

\section{CAS Managing Medicine in Health Care Organisations}

Medizinische Versorgung wird geplant, gestaltet, erbracht und bewirtschaftet. Dabei gilt es zahlreiche äussere wie innere Spannungen zu balancieren und dem missing link zwischen Effizienz und Effektivität auf die Spur zu kommen. Exakt das bedeutet Managing Medicine.

Ein gutes Verständnis der Möglichkeiten wie der Grenzen des Managements und seiner Instrumente zu haben, ist heute für medizinisches Führungspersonal absolut wesentlich. Dieses CAS an der medizinischen Fakultät der Universität Bern vermittelt zentrale Konzepte und Instrumente des Managements und der Steuerung medizinischer Organisationen. Ökonomisches und medizinisches Denken und Handeln werden dabei wechselseitig befragt, genutzt und in Beziehung gestellt.

Der Zertifikatskurs wendet sich an Personen aus dem medizinischen Kerngeschäft und vermittelt Wissen, Fähigkeiten und Haltungen, die es für erfolgreiches Gestalten von Gesundheitsorganisationen und ihren Bereichen heute braucht. Dies geschieht in einer inspirierenden und kollaborativen Lernumgebung.

Detaillierte Informationen unter www.cas-managingmedicine.ch 